\title{
Construction of Declaration and Audit network Platform for Experimental Instruction Equipment in Independent Colleges
}

\author{
Ya-Song LIU, Jie GAO, Jing-Guang WEI \\ ${ }^{1}$ Zhuhai College, Jilin University Zhuahi City, Zuhai, China \\ ${ }^{2}$ School of Continuing Education, Jilin University Changchun City, China. \\ jiegao26@163.com,921330@qq.com
}

Keywords: Declaration and audit network platform; Laboratory construction; Independent college

\begin{abstract}
It is essentially important to spend limited funds scientifically and efficiently on laboratory construction for independent colleges which focus on training practical talents. A network platform has been developed for declaring and auditing experimental instruction equipment in independent colleges. The application of this platform will make the procedure of the application and the evaluation of budgets more open, transparent, and avoiding repeated declare. Moreover, it enhances the preciseness of the demonstration in laboratory construction, which plays an important role in the construction of independent college.
\end{abstract}

\section{Introduction}

As the combination of public universities and private capital, independent colleges, by their nature, must give equal consideration to both economic and social benefits at the same time in the schoolrunning process. The school-running cost has always been an inevitable issue, and this is especially true at the initial stage of operation. Therefore, how to realize resources sharing, particularly the sharing of teaching resources, and how to maximize instruments utilization are also the critical problems for the school leaders to resolve [1-2]. Zhuhai College of Jilin University, as an independent college, was built in May of 2004, and the area of laboratories has been put to use by far, is about 50,000 square meters. Ever since we started on our laboratory construction planning, to ensure the scientific distribution of the laboratory construction funds, especially avoid unreasonable expenditures when making purchases of instruments has been made one of our top priorities so that resources sharing could be realized to its best advantage. To achieve this goal, a budget evaluation system for the laboratory construction was established, and several years' practice has proved its effect to be fairly satisfied. The self-developed "declaration and audit network platform for experimental instruction equipment" is an enssential factor that largely contributes to the successful implementation of this evaluation system.

\section{Background of Network Platform Development}

In order to further enhance the laboratory construction, our college has set up an experimental teaching and management center (hereafter refered to as experimental center) fully in charge of the planning, construction and management of the laboratories. During the preparation of the laboratory construction evaluation, the process of purchasing and auditing the selection of experimental instruction equipment is an important one that takes complex procedure and requires a great deal of patience. Since the equipment procurement in independent colleges so far, has not yet been included into the unified tender procedure of the government, it is in general, the investors' responsibility to organize the procurement according to the colleges' purchasing lists of the coming academic year. The experiment center, as the major organizer of the declaration and audit process, we insist on obtaining the purchase disposition from each academic unit in advance so as to see the limited fund utilizing and lab construction needs from a larger picture. Afterwards, the summary and audit processes are executed according to the collected filings from the academic units. During this procedure, we put much emphasis on increasing operation rate of the equipment for the realization of 
resource sharing while making sure the teaching quality is not in jeopardy. The workflow, as described above, one can imagine that some inevitable constraints must be resolved through consultation and coordination. Because of the multi-level communication and frequent discussion required for finalizing the plan, the traditional way of working with one teaching unit at a time would undoubtedly result in inefficiency. Aiming at resolving the above mentioned problems, we decided to develop a network platform to standardize the working procedures, cope with the time restriction, improve working efficiency, and achieve a paperless, rea-time online style of audit. This network platform breaks the very complex procedure into fractions and integrates them with the whole process of declaration and audit. In doing so, we are able to make audit and declaration happen at the same time, and eventually make the procedure of the application and the evaluation of budgets more open, transparent, and avoiding repeated declare.

\section{System Development and Major Functions and the Role of the Network Platform in Audit}

\section{System Development}

The system was constructed on the basis of B/S (Browser-Servier) architecture and J2EE threelayer (Struts + Spring + Hibernate) technology. By using MYSQL and MYSQL-FRONT database, the system was developed in the Eclipse Platform and operated in the Internet environment.

\section{The Client-side}

There are three major functions of the users' client: equipment declaration, declaration modification, and declaration query.

Equipment Declaration

Considering that besides of various types or models of the equipments, a huge amount of additional information for instance, the technical indices, or the use of special instruments is also required to be illustrated for equipment declaration, we have an "additional information" bar set up on the page for the users to fill in. Since other major data such as, desired purchase quality, reference price, and expected date of use is also required, the data volume may be extremely large, thus, the data entry cannot be done at once. Therefore, we also add a "save" button for the users to save their data regularly during the declaration process. The users can click on the "submit" button when all the data entry has been finished, and after this step, the declaration proceeds to the audit stage. In other words, the data that the users had just submitted is delievered to the staffs and concerned personnel from the experimental center via the network platform for an item-by-item review.

Declaration Modification

The users modify their data according to the review feedback. The audit process is a sophisticated task that often takes repeated modification to be finalized, and hence the feature of "declaration modification” definitely creates a friendly human-computer interface.

Declaration Query

The feature of "declaration query" is designed for the users' convenience to check their feedback updates regularly.

\section{Experimental Center's Reviewer-Side}

The review begins immediately after the users have had their data and relevant material submitted via the cilent-side. The major function of the reviewer-side of the experimental center includes declaration query, declaration audit, data and statistics, and statement printing.

Declaration query

Check declaration data in all statuses. Awaiting review, Returned and Passed are the three different statuses that the declaration data submitted by the users via the client-side may show depending on the stage of process it is currently at. The reviewers can check one or all three statuses simultaneously by clicking on different options. 


\section{Declaration Review}

To review the data that is awaiting audit. During the review process, the reviewers may initiate online discussion with the users about any relevant issuse, or leave opinions and/or comments in the "audit opinion" field if the users appear offline. Similarly, the users can leave offline messages for the reviewers as well.

For those users who have this system loaded but not yet logged on, once a feedback is sent by the reviewer-side, a tooltip window will pop up at the bottom right corner of the client-side once every 10 minutes. This pop-up window may last for 10 seconds each time, and will not stop showing until the users have logged on the system again.

Data and Statistics

Statistical analysis is conducted on the data that has passed the audit. This feature can also conduct analysis and comparison respectively on the users' data from previous years. Curve charts, pie charts, or histogram can be made to fulfill different requirements. We can accurately get hold of the investment that each department and teaching center have made in laboratory construction project by using the feature of "data and statistics". This function may also contribute to the future equipment replacement or upgrading by offering detailed evidence that can be referred to.

Print the Statement

Get the finalized statemtment printed in a unified format for further discussion and evaluation from the experts of the review committee.

Declaration switch bar

The switch bar is designed to set service hours of the platform. When it reaches the specified deadline of declaration, this network platform will automatically shut off, and no overdue information will be accepted in order to guarantee the seriousness and impartiality of the declaration process. However, manual intervention can be administered in some exceptional circumstances, to allow one or some users to make late submissions.

\section{The Role of the Network Platform in Audit}

\section{Improve Working Efficiency}

On the one hand, the staffs of the experimental center are able to review the information submitted and give feedback anytime and anywhere as long as the Internet is available. Without these restrictions of time and location, the working efficiency has been significantly improved. For the staffs from the education units, on the other hand, are able to submit their plans and data at any time, and communicate their questions about the feedback timely with the staffs of the experimental center online. This efficient way of interaction has effectively ensured their procurement to be completed on schedule.

\section{The Transparency of Declaration}

Real-time declaration and audit can be implemented in the true sense for the entire process is completed via the Internet platform; therefore, enables the experimental center to master the relevant information of each teaching unit in a timely and exhaustive manner. Similarly, each teaching unit can also make inquiries via the client, and this clearly, has the transparency improved. Meanwhile, the experimental center is able to make horizontal adjustment according to the given data readily, to gain a better overall balance among the units for fully practicing the principle of resource sharing.

\section{Enhance the Preciseness of Review Process}

Before the declaration and audit network platform for experimental instruction equipment has come into service, declaration of each application cannot be started until all the data and relevant material have been collected. For this reason, some teaching units fail to submit their material by deadline only for a small portion of the required material has not been integrated in time. In this case, not only the entire agenda of declaration and audit is interrupted, but also severely limits the time that the experimental center ought to invest in the process of declaration and audit. With such a huge 
workload, the chance of flaws and errors appearing during the declaration and audit process can be largely increased for the experts from the review committee have to finish the work within such a short period of time. Beyond all doubt, the experts' workload would be dramatically reduced and therefore, be able to focus on solving the remaining problems, if we had done a well-organized and adequate preparatory job before hand. In this way, experts' valuable expertise can be more effectively distributed in examining the technical index and analyzing equipment' model selection.

\section{Conclusion}

Laboratory construction is a comprehensive project which heavily involves human resources, funds, and equipment. Therefore, long-term strategic plannings must be made in accordance with the running principle of college for laboratory constructions. That is, to establish a vision of development by taking various kinds of objective and subjective factors, such as goals and means, technology and economy, advancement and feasibility, and both current and future needs in to fully consideration for the optimal investment returns and construction effects [7-11]. The application of the declaration and audit network platform for experimental instruction equipment on the one hand, elevates the effectiveness of the process of declaration and audit of the teaching instrument and equipment purchase and the preciseness of laboratory construction evaluation. On the other hand, this platform, as an integral part of our laboratory construction budget system, plays an important role in making the laboratory construction process more scientific and transparent.

\section{References}

[1] Bing Fu, Discussion on the laboratory construction plan of the Independent Institute [J]. Science of laboratory. vol 4, pp. 150-152, 2011.

[2] Ji-na Zhou, Control on the risk in equipment purchase of the Independent Institute [J]. Science of laboratory. vol 5, pp. 188-190, 2011.

[3] Hai-lin Jia, Li-gang Zheng, Analysis and Discussion on laboratory construction [J]. Science of laboratory. vol 1, pp. 136-138, 2010.

[4] Shi-hong Cheng, Thought and practise of construction of labratory center in universities[J]. Science of laboratory. vol 4, pp. 120-123, 2012.

[5] Jie Zhu, Problem and strategy existing in laborary construction in mutiple colleges [J]. Science of laboratory. vol 2, pp. 152-153, 2011.

[6] E-na Cai, Lv-si Xu, Exploration and practise of sharing platforms and management model [J]. Laboratory research and exploration. vol 3, pp. 377-379, 2011.

[7] Han-ying Chen, Chao Hou, Strengthening the laboratory construction is the only way to develop independent colleges [J]. Laboratory research and exploration. vol 29(9), pp. 171-172, 2010.

[8] Sheng-li Xie, Jian-xia Gou, Universities improve equipment utilization, training applied talent [J]. Laboratory research and exploration. vol 30(11), pp. 411-413, 2011.

[9] Chun-hui Li, Study and Practice on talents training programme of network system management [J].China Educational Technique \& Equipment. Vol 8, pp. 55-56, 2012.

[10] Yan Xue, Universities Application of Modern Educational Technology Practice [J]. China Educational Technique \& Equipment. Vol 18, pp. 11-13, 2012.

[11] Hai-lian Kou, Zheng-gang Wan, Teacher Professional Development Strategy based video case under the Network Environment [J]. China Educational Technique \& Equipment. Vol 18, pp. 19-20, 2012 